\title{
LA AUTONOMÍA ECONÓMICA DE LAS MUJERES PARA UNA RECUPERACIÓN TRANSFORMADORA CON IGUALDAD EN AMÉRICA LATINA Y EL CARIBE
}

\begin{abstract}
La desigualdad de género es históricamente un rasgo estructural en América Latina y el Caribe que limita la autonomía económica de las mujeres y se expresa en una distribución desigual entre mujeres y hombres del poder, los recursos, la riqueza, el trabajo y el tiempo. Este trabajo analiza el impacto de la crisis provocada por la pandemia del COVID-19, en la autonomía económica de las mujeres en América Latina y el Caribe. El texto concluye con medidas de corto, mediano y largo plazo en diferentes sectores y áreas, orientadas a una recuperación transformadora con sostenibilidad y con igualdad, desde los aportes de la Comisión Económica para América Latina y el Caribe.
\end{abstract}

\section{Women' s economic autonomy for a transformative recovery with equality in Latin America and the Caribbean}

Gender inequality is historically a structural feature in Latin America and the Caribbean that limits women's economic autonomy and is expressed in an unequal distribution between women and men of power, resources, wealth, work and time. This paper analyzes the impact of the crisis caused by the COVID-19 pandemic on women's economic autonomy in Latin America and the Caribbean. The text concludes with short, medium and long term measures in different sectors and areas, aimed at a transformative recovery with sustainability and equality, based on the contributions of the Economic Commission for Latin America and the Caribbean.

Palabras clave: igualdad de género, trabajo remunerado, trabajo no remunerado, economía del cuidado, recuperación transformadora.

Keywords: gender equality, paid work, unpaid work, care economy, transformative recovery.

JEL: D63, E24, J16, O15.

\footnotetext{
* Secretaria Ejecutiva de la CEPAL (Comisión Económica para América Latina y el Caribe).

Las opiniones expresadas en el presente artículo no representan necesariamente las de la Organización. Los derechos de autor del presente artículo pertenecen a la Organización de las Naciones Unidas. La Organización autoriza el uso del presente artículo con fines exclusivamente académicos, para su publicación, en formato digital e impreso, en el monográfico número 921 de Información Comercial Española, Revista de Economía y para cualquier eventual uso ulterior, en parte o en la totalidad del artículo, debe solicitarse la respectiva autorización a la CEPAL (publicaciones.cepal@cepal.org).

La Secretaria Ejecutiva desea agradecer a la señora María Lucía Scuro, Oficial de Asuntos Sociales y a la señora Belén

Villegas, Consultora, ambas de la División de Asuntos de Género de la CEPAL, por sus contribuciones a este artículo.

Contacto: SECRETARIA.EJECUTIVA@cepal.org

Versión de junio de 2021.

https://doi.org/10.32796/ice.2021.921.7264
} 


\section{Introducción}

Las crisis sanitaria, social y económica acontecidas a escala mundial han impuesto desafíos que son globales; sin embargo, la resiliencia de los países y regiones a esta crisis dista mucho de ser homogénea. En 2019, América Latina y el Caribe cerraba el año con un muy bajo crecimiento regional de solo $0,1 \%$. De hecho, el escenario macroeconómico regional mostraba para el período 2014-2019 una desaceleración generalizada de la actividad económica, caídas del PIB per cápita, la inversión, el consumo per cápita y las exportaciones, un menor volumen en el comercio y un sostenido deterioro de la calidad del empleo (CEPAL, 2019a).

Las brechas estructurales de la región, su limitado espacio fiscal, el débil acceso a la protección social, la elevada informalidad laboral, la heterogeneidad estructural, entre otros factores de largo plazo, han determinado el alcance de los efectos de la pandemia en las economías regionales. En este escenario, comparando diferentes indicadores sanitarios, económicos y sociales, América Latina y el Caribe se presenta como la región más afectada del mundo emergente (CEPAL, 2020a).

La caída del PIB regional $(-7,1 \%)$ ha impactado significativamente en los niveles de empleo y en los ingresos de los hogares en todos los países, al tiempo que ha acentuado las desigualdades ${ }^{1}$. En efecto, la actual pandemia ha hecho aún más visible el carácter estructural de las desigualdades de género en América Latina y el Caribe y la fuerte exposición de las mujeres a los impactos de la crisis porque al igual que la desigual distribución de los beneficios del crecimiento,

\footnotetext{
${ }^{1}$ La CEPAL ha creado recientemente el Observatorio COVID-19 para dar seguimiento a las medidas adoptadas por los países para enfrentar y mitigar los efectos de la pandemia e incluir una sección específica sobre igualdad de género. El Observatorio también ofrece análisis de las repercusiones económicas y sociales que tendrán estas políticas a nivel nacional y sectorial. Se han elaborado informes e historias políticas basadas en datos, tanto a nivel regional como nacional.
}

los costos de los ajustes económicos y las crisis también se materializan de manera desigual.

La crisis provocada por la pandemia del COVID-19 implicó amplias caídas en la ocupación y en las condiciones laborales de las mujeres en América Latina y el Caribe, generando un retroceso de más de una década en los avances logrados en materia de participación laboral. Además, esta crisis intensificó la carga de trabajo no remunerado de los hogares, poniendo en evidencia la injusta organización social de los cuidados. De esta manera, la pandemia ha profundizado los nudos estructurales de la desigualdad, atentando gravemente contra la autonomía económica de las mujeres (CEPAL, 2021a).

La pandemia ha transformado los problemas crónicos del estilo de desarrollo de la economía mundial en un cuadro agudo que requiere una respuesta inmediata. La crisis puede, en este sentido, ser una oportunidad para impulsar una nueva trayectoria en el estilo de desarrollo en la región permitiendo avanzar hacia el logro de la autonomía de las mujeres en su diversidad y la igualdad de género. La autonomía de las mujeres es, a la vez, una condición de un futuro con igualdad y sostenibilidad.

\section{Los efectos de la crisis en la autonomía económica de las mujeres}

La desigualdad de género ha sido un rasgo estructural en América Latina y el Caribe que limita la autonomía de las mujeres. La desigualdad socioeconómica y la persistencia de la pobreza; los patrones culturales patriarcales discriminatorios y violentos y el predominio de la cultura del privilegio; la rígida división sexual del trabajo y la injusta organización social del cuidado, y la concentración del poder, constituyen los nudos estructurales definidos en el marco de la Conferencia Regional sobre la Mujer de América Latina y el Caribe. Estos nudos, sobre los que se sustenta un modelo de desarrollo que ya ha probado ser insostenible, se refuerzan entre sí limitando el pleno goce de los 
derechos de las mujeres y el avance hacia la igualdad sustantiva (CEPAL, 2017).

La heterogeneidad estructural de los países de la región se refuerza con la actual división sexual del trabajo, determinando interacciones recíprocas entre la posición ocupada por las mujeres en el trabajo remunerado y el trabajo no remunerado (CEPAL, 2019b). De esta manera, las mujeres asumen la mayor parte del trabajo (no remunerado) que permite la sostenibilidad de la vida al tiempo que exhiben una menor participación en el empleo, concentración en sectores económicos y ocupaciones de menor productividad, menores niveles de ingresos y el consecuente bajo acceso a la protección social.

La crisis por COVID-19 está profundizando los nudos estructurales de la desigualdad de género, visibilizando fisuras en los sistemas sociales, políticos y económicos a través de la disminución de la participación laboral de las mujeres y la intensificación de la demanda de cuidados. En términos generales, la crisis ha tenido graves efectos en la región y, especialmente, en los niveles de actividad, empleo e ingresos de las mujeres. Se observa un deterioro de las condiciones laborales, en particular una reducción general de la participación laboral, así como una concentración de las mujeres en sectores más expuestos a la pérdida de empleo y de ingresos (CEPAL, 2019b).

Esta situación amenaza los avances logrados en décadas anteriores. De hecho, entre los años 1997 y 2007, la tasa de participación de las mujeres se incrementó 5,3 puntos porcentuales (Vaca-Trigo, 2019). Sin embargo, desde entonces ha aumentado levemente, hasta alcanzar un promedio del $51,3 \%$ en 2019 , 22,7 puntos porcentuales por debajo de la de los hombres, tendencia que se ha agudizado en el escenario actual; entre el segundo trimestre de 2019 y el mismo período de 2020, hubo una pérdida inédita de puestos de trabajo que afectó en mayor medida a las mujeres que a los hombres (CEPAL/OIT, 2020). Así, en el año 2020, la tasa de desocupación de las mujeres proyectada sobre los niveles de ocupación de 2019 alcanza un $22,2 \%$ en 2020 , lo que implica 12,6 puntos porcentuales de variación interanual (CEPAL, 2020a).

Durante el tercer trimestre del año 2020, la situación mejoró levemente y en casi todos los países se detuvo la caída del nivel del empleo y el número de personas ocupadas aumentó respecto al trimestre anterior. Sin embargo, esta leve recuperación se concentró en el empleo masculino. Así, en el conjunto de 11 países de la región, el número de hombres ocupados aumentó un $7,1 \%$ con respecto al trimestre anterior, mientras que el número de mujeres ocupadas se incrementó tan solo en un $4,1 \%$ (ibid, pág. 81 ).

\section{Los sectores más afectados}

Además de los efectos a nivel agregado en la economía, se observan efectos diferenciados en los sectores, cuya magnitud dependerá de la combinación de factores externos con las características internas de las economías de cada país. Las mujeres se concentran en sectores como el comercio, el turismo, la industria manufacturera y en sectores de la economía del cuidado (empleo doméstico, salud y enseñanza) (CEPAL, 2019b) que, en el actual escenario, se han visto tensionados de múltiples maneras.

Las medidas adoptadas para frenar los contagios han afectado en mayor medida a algunos sectores de la economía, que por sus características presentan mayores riesgos en términos del volumen de la producción y de la pérdida del empleo (CEPAL/OIT, 2020). En particular, el turismo, el comercio y la manufactura son sectores especialmente expuestos a los canales de transmisión externos de la crisis, como el cierre total o parcial de las fronteras, la reducción de la demanda de turismo y la interrupción de las cadenas globales de valor (Figura 1). Estos sectores, junto con el empleo doméstico, han sido precisamente los que sufrieron mayores pérdidas en términos de volumen de la producción y empleo (Bidegain Ponte et al., 2020). El 56,9 \% del empleo de las mujeres y el $40,6 \%$ del empleo de los hombres en América Latina se concentra en sectores de 
FIGURA 1

\section{AMÉRICA LATINA Y EL CARIBE (17 PAÍSES) CARACTERÍSTICAS DE SECTORES EN RIESGO ALTO Y BAJO, CON ALTA PRESENCIA DE MUJERES, PROMEDIOS PONDERADOS, ALREDEDOR DE 2019}

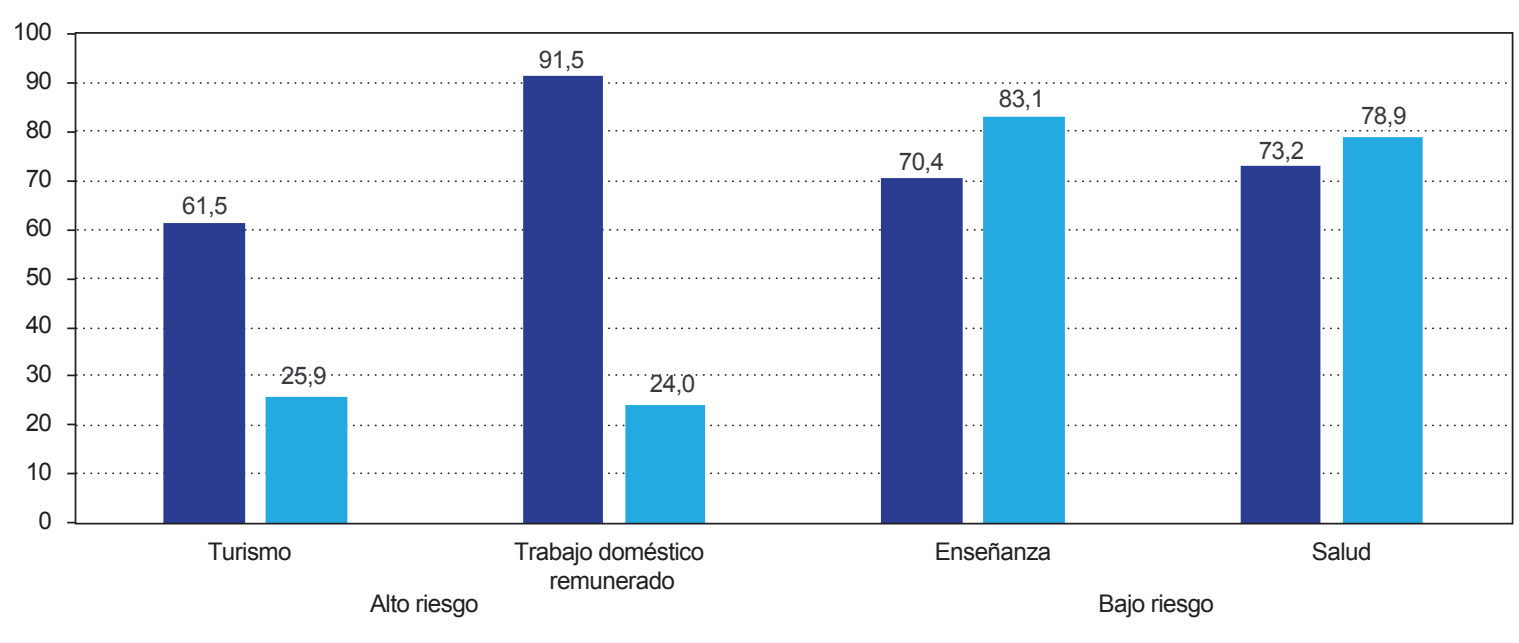

a Proporción de mujeres a Proporción de mujeres afiliadas o cotizantes a la Seguridad Social

FUENTE: Comisión Económica para América Latina y el Caribe (CEPAL), sobre la base de Banco de Datos de Encuestas de Hogares (BADEHOG).

alto riesgo económico y de pérdida de empleo, mientras en el Caribe estas cifras son de $54,3 \%$ del empleo femenino y $38,7 \%$ del masculino (CEPAL, 2021a).

Algunos de los sectores que se encuentran en mayor riesgo se caracterizan, además, por altas tasas de informalidad, bajas remuneraciones y bajos niveles de cualificación. De hecho, siguiendo la información contenida en CEPAL (2021a), un bajo porcentaje de mujeres en estos sectores están afiliadas o cotizan a un sistema de Seguridad Social, a saber: un 35,8 \% en el sector del comercio, un $45,4 \%$ en el sector de las manufacturas, un $25,9 \%$ en el sector del turismo y un $24,0 \%$ en el sector de los hogares como empleadores.

Además, la industria manufacturera se ha visto especialmente afectada por las interrupciones en las cadenas globales de suministro y el cierre de fábricas. Esto puede ser particularmente crítico en Centroamérica, donde un $13,2 \%$ del empleo femenino se concentra en este sector, con una gran participación en las industrias orientadas a la exportación, como la maquila y el ensamblaje. Los cierres de estas industrias pueden provocar disrupciones en las trayectorias laborales de las mujeres, que suelen tener bajos niveles de cualificación y que verán, en consecuencia, dificultades para reinsertarse laboralmente.

El sector del comercio se caracteriza por concentrar una proporción importante del empleo femenino (un $21,6 \%$ de las ocupadas) y -a diferencia de la industria manufacturera - una elevada proporción de empleo informal. Un 69,0 \% de estas mujeres se concentran en empresas de menos de cinco personas y un $41,1 \%$ son trabajadoras por cuenta propia.

La menor actividad económica ha impactado sustancialmente en los niveles de ventas y el empleo 
en el comercio, provocando el cierre de aproximadamente 2,7 millones de microempresas formales en la región (CEPAL, 2021a). En este contexto, las mujeres se han visto mayormente afectadas debido a que se concentran en el segmento de empresas más expuestas a la crisis como son las empresas pequeñas y medianas. Por otra parte, la imposibilidad de llevar a cabo relaciones presenciales con los clientes, que suelen muchas veces requerirse en el trabajo por cuenta propia, y las barreras estructurales de género en materia de acceso al crédito, hacen aún más difícil la resistencia y recuperación de las mujeres cuentapropistas $^{2}$ (CEPAL, 2020a).

El sector de las actividades de alojamiento y de servicio de comidas (asociadas al turismo) también es un sector altamente feminizado $(61,5 \%$ de los puestos de trabajo estaban ocupados por mujeres en el año 2019). Al igual que el comercio, aquí también el empleo de las mujeres se concentra en microempresas (un 69,5\% de las mujeres en este sector son empleadas en empresas de menos de cinco personas) (CEPAL, 2021a).

Si bien los efectos económicos y sociales de la crisis sobre el turismo son notorios en todos los países de la región, lo son particularmente en el Caribe donde la contracción de las economías de esta subregión tiene estrecha relación con la reducción de la demanda de servicios turísticos. En la subregión del Caribe, el sector del turismo representa un $11,9 \%$ del empleo femenino y un $5,7 \%$ del empleo masculino, siendo incluso mayor en algunos países como Santa Lucía $(20,8 \%)$, Barbados (16,2\%) y Jamaica (11,9\%), ibid.

El trabajo doméstico remunerado es otro sector especialmente afectado por la crisis debido a la imposibilidad de ser realizado de manera remota y a ser un sector muy precarizado en sus condiciones laborales. En América Latina y el Caribe, a 2019, alrededor de 13 millones de personas se dedicaban al trabajo doméstico remunerado, siendo el 91,5\% mujeres, muchas de ellas afrodescendientes, indígenas y/o

\footnotetext{
2 Trabajadoras por cuenta propia o trabajadoras autónomas.
}

migrantes (CEPAL, 2021b). Este sector está altamente precarizado: sus salarios se encuentran entre los más bajos del conjunto de trabajadores remunerados y sus niveles de informalidad son especialmente altos (76 \% de las mujeres que allí se emplean no cuentan con cobertura previsional) (Valenzuela et al., 2020).

En América Latina, el 51,6 \% de las personas que migran son mujeres $y$, de este conjunto, más de un tercio está ocupado en el trabajo doméstico remunerado $(35,3 \%)$. El cierre de fronteras y el impacto en el empleo en este sector ha provocado la disminución de salarios o la pérdida de empleos, afectando no solo a las condiciones de vida de estas mujeres sino también el ingreso de los hogares que dependen en gran medida de las remesas generadas por ellas. Esto sucede, en particular, en países como los de Centroamérica, donde los cuidados suelen costearse con las remesas enviadas por trabajadoras domésticas que se desempeñan en otro país diferente al cual reside su familia, conformando parte de lo que se ha dado en llamar como "cadenas globales de cuidado» (OIT, 2016).

Más del $99 \%$ de las trabajadoras domésticas remuneradas de la región viven en países que han establecido restricciones de movimiento para evitar mayores contagios. El grado de confinamiento y su duración, junto con el nivel de informalidad del sector en cada país, explican la severidad del impacto de la pandemia en el empleo y los ingresos de las trabajadoras domésticas remuneradas en cada caso (Valenzuela et al., 2020). Más allá de las especificidades, en términos generales, el $69 \%$ de las trabajadoras domésticas remuneradas de América Latina y el Caribe se han visto afectadas en la pérdida de sus empleos o la reducción de sus jornadas de trabajo, siendo esta cifra 20 puntos porcentuales más alta que la cifra del promedio mundial (49 \%) (OIT, 2020).

El impacto de la pandemia en el empleo no solo se manifiesta en el aumento de la desocupación, sino también en la intensificación del trabajo en algunos sectores en particular, tales como la salud y la educación. 
En el caso del sector salud, donde las mujeres alcanzan el $73,2 \%$ del total de las personas empleadas (CEPAL, 2021a), la respuesta a la crisis en la primera línea de atención ha implicado un aumento de las jornadas laborales y mayor exposición al contagio, que en algunos casos se ha agravado debido a la insuficiencia de equipos de protección, falta de personal y saturación de la infraestructura sanitaria.

En el caso de la educación, donde el 70,4 \% de los puestos de trabajo son ocupados por mujeres, las jornadas de trabajo y las tareas asociadas al ejercicio docente se han complejizado y ampliado. EI cuerpo docente ha debido responder a las nuevas formas de educación, en muchos casos sin la posibilidad de formación y sin los recursos necesarios para poder adaptar su trabajo a las exigencias de la enseñanza a distancia. Además, han sido frecuentes los casos donde el personal de la educación ha desempeñado tareas orientadas a asegurar las condiciones de seguridad material de las y los estudiantes y sus familias, tales como la distribución de alimentos, productos sanitarios y materiales escolares, entre otras actividades conexas a la mitigación de la crisis (CEPAL, 2021a).

\section{La economía del cuidado esencial para la sostenibilidad de la vida}

La economía del cuidado comprende las relaciones entre el trabajo de cuidados remunerado que se realiza en el mercado laboral y el no remunerado que se realiza en el seno de los hogares (CEPAL, 2019b). Dada la actual división sexual del trabajo, todos los sectores que componen la economía del cuidado son altamente feminizados, siendo este un sector sostenido esencialmente por el trabajo de las mujeres.

Las actividades de trabajo no remunerado en el ámbito de los hogares incluyen una amplia gama de tareas, entre ellas la limpieza y el mantenimiento del hogar, la preparación de alimentos, la realización de compras y el apoyo en tareas de higiene personal, educativas y de acompañamiento a miembros del hogar o familiares, entre otras tareas. Por su parte, el trabajo remunerado de la economía del cuidado puede descomponerse en cuatro categorías. Estas categorías son: i) las personas que trabajan en tareas de cuidado de forma remunerada en los sectores de educación, asistencia social y salud; ii) personas en ocupaciones no relacionadas con el cuidado empleadas en los sectores de educación, asistencia social y salud (funcionarios administrativos, cocineros, limpiadores, etc.); iii) personas que trabajan en el sector de los hogares particulares (trabajadoras domésticas remuneradas); y iv) personas empleadas en otros sectores que ejercen tareas de cuidado de forma remunerada (CEPAL, 2019b).

Este grupo amplio y heterogéneo difiere en cuanto a las ocupaciones que desempeña, las habilidades requeridas, las condiciones en el empleo y los niveles de remuneración. Sin embargo, la división sexual del trabajo determina que, frecuentemente, todas estas actividades suelan asociarse con habilidades que suelen atribuirse a la condición «natural» de las mujeres y, en consecuencia, se conciben en general como actividades de baja cualificación. Por lo tanto, estas labores en el mercado laboral son desempeñadas en su mayoría por mujeres y suelen caracterizarse por altos niveles de precariedad en materia de calidad del empleo y bajos salarios (OIT, 2018).

La división sexual del trabajo y la injusta organización social del cuidado, persisten como nudos estructurales de la desigualdad en América Latina y el Caribe. Ambas dimensiones interactúan mutuamente atentando contra el pleno ejercicio de los derechos de las mujeres y su autonomía, y generando además una serie de ineficiencias económicas y sociales, con externalidades negativas para el conjunto de la sociedad (CEPAL, 2021b).

El tipo de oferta, los niveles de empleo y condiciones laborales del sector de los cuidados remunerados incide, significativamente, en la calidad de los servicios de cuidado. A su vez, la oferta de servicios públicos y privados, accesibles y de calidad, permite redistribuir la responsabilidad sobre la realización del trabajo de 


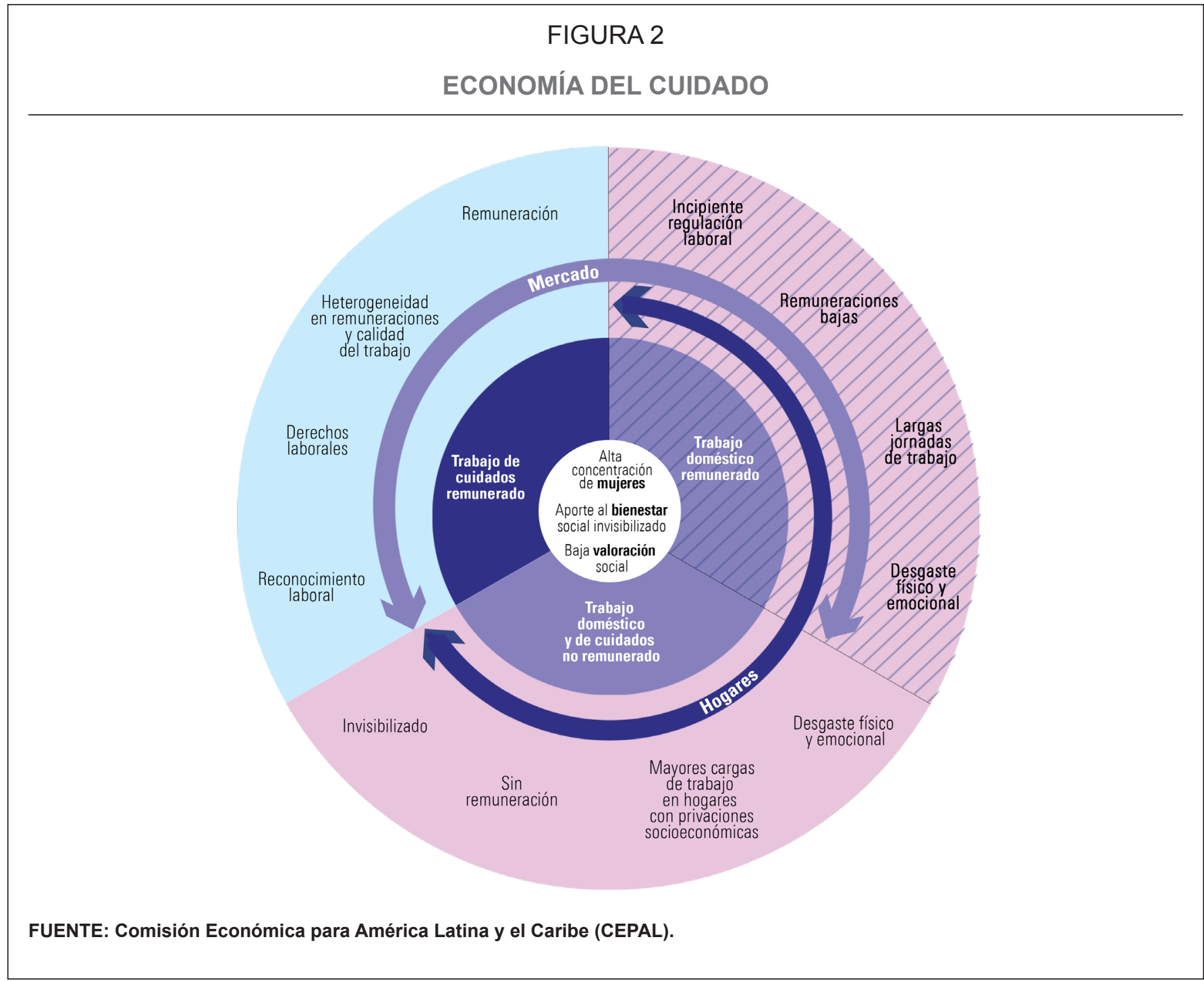

cuidados desde los hogares hacia el Estado y el sector privado, liberando tiempo de las mujeres para realizar otras tareas de esparcimiento, capacitación, movilización y activismo, etc.; todo lo cual impacta en su bienestar general y su autonomía económica.

De esta manera, la economía del cuidado (Figura 2) incluye las labores fundamentales para la reproducción de la vida que realizan los hogares, las dinámicas de los sectores de mercado que brindan cuidados (calidad del empleo y la prestación de servicios), la provisión de infraestructura orientada al cuidado y la formulación de políticas públicas, así como la interrelación recíproca entre estas esferas y sectores (ibid $)^{3}$.

Con anterioridad a la crisis sanitaria del COVID-19, en los países de la región sobre los que se dispone de datos, las mujeres destinaban a las actividades de trabajo doméstico y de cuidados entre 22 y 42 horas semanales; el triple del tiempo que dedican los hombres a las mismas tareas (CEPAL, 2020b). Además, esta distribución presenta diferentes valores según el nivel de

3 Ídem. 
ingresos: las mujeres pertenecientes a los hogares del quintil de menores ingresos dedican alrededor de un $39 \%$ más de horas semanales al trabajo no remunerado que las pertenecientes a los hogares del quinto quintil de ingresos (CEPAL, 2021b). De hecho, el principal obstáculo para la plena inserción de las mujeres en el mercado laboral está relacionado con las responsabilidades familiares, expresadas en el trabajo doméstico y de cuidados; alrededor de un $60 \%$ de las mujeres en hogares con presencia de niños y niñas menores de 15 años declara no participar en el mercado laboral por atender responsabilidades familiares, mientras que en hogares sin presencia de niños y niñas del mismo tramo de edad, esta cifra se ubica en el $18 \%$ (ibid).

Las medidas de confinamiento han llevado a la sobrecarga del trabajo de cuidados en los hogares, agudizando las brechas de género. Además, en este escenario donde los sistemas sanitarios están operando al máximo de sus capacidades, parte de la atención de la salud ha sido trasladada a los hogares, lo que ha aumentado aún más el trabajo de cuidados en los hogares, fundamentalmente a cargo de las mujeres. En efecto, en el año 2020, se registró una significativa salida de mujeres de la fuerza laboral, quienes teniendo que atender las demandas de cuidados en sus hogares, no retomaron la búsqueda de empleo (CEPAL, 2021a).

Esta injusta distribución del trabajo de cuidados impacta, notoriamente, en la autonomía económica de las mujeres en la medida de que largos períodos fuera del mercado laboral pueden entrañar un deterioro de las trayectorias laborales y, por ende, un deterioro de los ingresos presentes y futuros, así como de la cobertura en materia de Seguridad Social y derechos jubilatorios.

Las tareas relativas al trabajo de cuidados a pesar de ser esenciales para la vida no son valoradas $y$, por ende, no se les atribuye un valor monetario. Sin embargo, los cálculos acerca de la valorización económica del trabajo no remunerado de los hogares realizados en los países de América Latina y el
Caribe, señalan que este trabajo representaría entre el $15,7 \%$ y el $24,2 \%$ del PIB y que son las mujeres quienes aportan cerca del $75 \%$ de este valor (CEPAL, 2021c).

La economía del cuidado debería ser pensada como una inversión en un sector que puede ser clave en la reactivación para la salida de la crisis. Los servicios de cuidado suelen ser incluidos en la partida de gasto social al momento del diseño presupuestal. Sin embargo, se ha encontrado que los recursos que se destinan a la economía del cuidado constituyen más bien una inversión y tienen un impacto positivo en el resto de la economía (Braunstein et al., 2011). La inversión en la economía del cuidado redunda en una mayor calidad y cantidad de empleos en sectores tradicionalmente feminizados, aumentando el nivel de ingresos de quienes se emplean en estos sectores (CEPAL, 2021b). Este aumento del ingreso impacta, asimismo, en la capacidad de consumo de los hogares, lo que a su vez mejora la actividad económica y permite que una parte de lo invertido vuelva en forma de recaudación (ibid).

Además, a mediano y largo plazo, la inversión en los sectores de cuidado impacta positivamente en la educación de la fuerza de trabajo y, por lo tanto, en su productividad (Figura 3). Asimismo, la provisión de infraestructura pública y social de cuidado puede reducir las desigualdades educativas que afectan a niñas y niños, especialmente si se regula y controla la calidad de los servicios comunitarios, públicos y privados que brindan cuidados. De esta manera, la inclusión de la economía del cuidado en los planes de mitigación y reactivación de la crisis del COVID-19, no solo dinamizaría las economías, sino que constituye una herramienta central para una recuperación transformadora, que sea sostenible y que mejore los niveles de igualdad (CEPAL, 2021c).

En términos de la Agenda 2030 para el Desarrollo Sostenible, la inversión en la economía del cuidado contribuye, entre otras cosas, a eliminar la pobreza e implementar sistemas y medidas apropiados de 


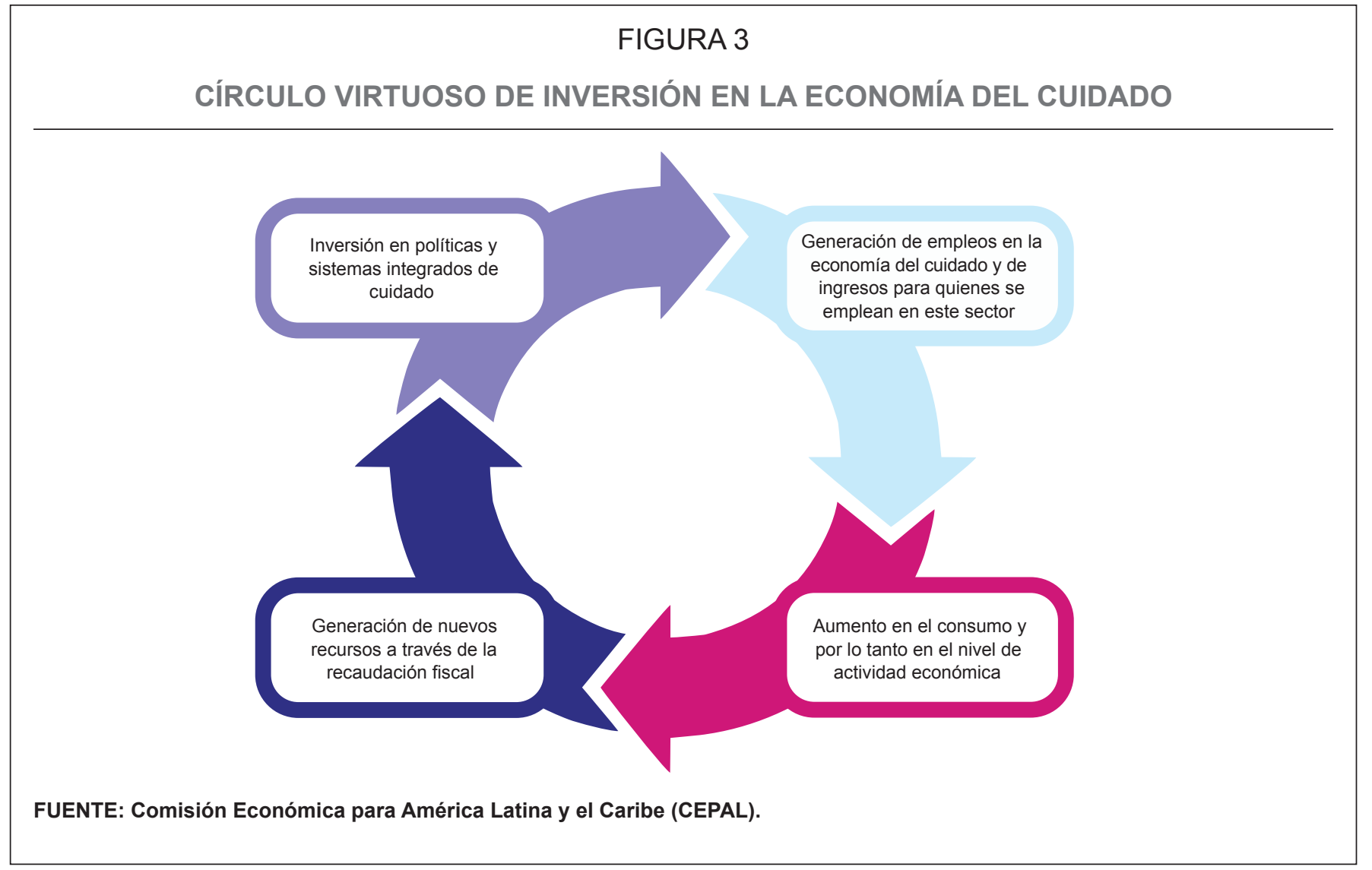

protección social para todas las personas (ODS 1), garantizar una vida sana (ODS 3 ), lograr la igualdad de género (ODS 5), promover el crecimiento económico inclusivo y sostenible (ODS 8 ) y reducir las desigualdades (ODS 10).

En el marco regional, desde el año 1977, América Latina y el Caribe se constituye como la única región del mundo donde, durante más de cuatro décadas y de manera ininterrumpida, los Estados se reúnen para debatir y comprometerse políticamente a erradicar la discriminación hacia las mujeres. Esto permite el avance en una Agenda Regional de Género (ARG) en sinergia con compromisos vinculantes como la Convención sobre la eliminación de toda forma de discriminación contra la mujer (CEDAW, por sus siglas en inglés), la Convención de Belém do Pará y, desde luego, con la Agenda 2030 para el Desarrollo Sostenible y sus Objetivos de Desarrollo Sostenible (ODS). A su vez, la Agenda Regional de Género es complementada con otros acuerdos regionales de los Gobiernos asumidos en la Conferencia Regional sobre Población y Desarrollo ${ }^{4}$, la Conferencia Estadística de las Américas ${ }^{5}$ y la Conferencia Regional de Desarrollo Social ${ }^{6}$.

Reforzando lo acordado en la meta 5.4 de los ODS que propone reconocer y valorar los cuidados y el trabajo doméstico no remunerado, los mecanismos para el adelanto de las mujeres de Gobiernos de América Latina y el Caribe reunidos en el marco de la Conferencia Regional sobre la Mujer, renuevan el compromiso de

\footnotetext{
${ }^{4}$ Conferencia Regional sobre Población y Desarrollo de América Latina y el Caribe.

${ }^{5}$ Conferencia Estadística de las Américas.

${ }^{6}$ Conferencia Regional sobre Desarrollo Social de América Latina y el Caribe.
} 
superar la división sexual del trabajo y avanzar hacia una mejor distribución del trabajo (remunerado y no remunerado) entre hombres y mujeres, y promoviendo la corresponsabilidad entre el Estado, el mercado, la comunidad y las familias para la provisión de los cuidados necesarios para la vida de todas las personas.

Esto se expresa, por ejemplo, en el Consenso de Quito $(2007)^{7}$ aprobado durante la X Conferencia Regional sobre la Mujer de América Latina y el Caribe, donde se reconoce el valor social y económico del trabajo doméstico no remunerado que, sobre la base de la división sexual del trabajo, realizan fundamentalmente las mujeres. Además, se reconoce al cuidado como un asunto público que compete a los Estados desde donde se debe favorecer la responsabilidad compartida entre mujeres y hombres superando los estereotipos de género.

En esta misma línea, la Estrategia de Montevideo para la Implementación de la Agenda Regional de Género en el marco del Desarrollo Sostenible hacia $2030^{8}$, aprobada durante la XIII Conferencia Regional sobre la Mujer de América Latina y el Caribe (2016), sostiene que la división sexual del trabajo y la injusta organización social del cuidado constituye uno de los nudos estructurales de la desigualdad. Por ello, se plantea la necesidad de responder a las demandas de cuidado desde una perspectiva de corresponsabilidad entre hombres y mujeres, y entre las diversas formas de familia, las organizaciones sociales y comunitarias, las empresas y el Estado en la provisión de los cuidados.

Continuando y fortaleciendo el acumulado previo, en enero de 2020, se adoptó el Compromiso de Santiago en la decimocuarta sesión de la Conferencia Regional sobre la Mujer de América Latina y el Caribe. En esta oportunidad, se acordó: medir los efectos multiplicadores del impulso a la economía del cuidado, diseñar sistemas integrales de cuidados,

\footnotetext{
${ }^{7}$ Consenso de Quito, 2007.

${ }^{8}$ Estrategia de Montevideo, 2016.
}

y promover medidas, políticas y programas para el reparto equitativo del trabajo doméstico y de cuidado entre hombres y mujeres ${ }^{9}$.

Adicionalmente, en febrero de 2021, durante la 60. ${ }^{a}$ Reunión de la Mesa Directiva de la Conferencia Regional sobre la Mujer de América Latina y el Caribe ${ }^{10}$, en conjunto con la sesión especial de consulta regional previa al $65 .^{\circ}$ período de sesiones de la Comisión de la Condición Jurídica y Social de la Mujer, los gobiernos acordaron trabajar en la generación de un Pacto por los Cuidados, que luego fue retomado en el marco del Foro Generación Igualdad en la forma de una Alianza regional para los cuidados.

\section{Medidas transformadoras para una recuperación con igualdad}

La pandemia ha evidenciado los problemas estructurales de desarrollo regional, así como los nudos estructurales de la desigualdad, y en especial de la desigualdad de género, visibilizando fisuras en los sistemas sociales, políticos y económicos. En este contexto, es necesario implementar, por un lado, medidas de carácter urgente orientadas a la mitigación y recuperación de los efectos de la crisis y, por otro lado, implementar acciones orientadas a transformar las condiciones estructurales de la desigualdad con una visión de futuro. La crisis puede, en este sentido, ser una oportunidad para impulsar una nueva trayectoria en el estilo de desarrollo en la región permitiendo avanzar hacia el logro de la autonomía de las mujeres.

En el corto plazo, es necesario transversalizar la perspectiva de igualdad de género en todas las políticas de mitigación y recuperación de la crisis, incentivando además la participación de las mujeres en toda su diversidad, en roles de liderazgo y en los espacios de toma de decisiones de estos planes. La transversalidad de la

\footnotetext{
${ }^{9}$ Compromiso de Santiago, 2020.

10 Sexagésima Reunión de la Mesa Directiva de la Conferencia Regional sobre la Mujer de América Latina y el Caribe.
} 
perspectiva de género en todas las políticas de recuperación es necesaria pero no suficiente. Se requieren, además, acciones afirmativas en el ámbito del empleo, la fiscalidad, las políticas productivas, económicas y sociales que protejan los derechos de las mujeres, que eviten retrocesos y que enfrenten las desigualdades de género en el corto, mediano y largo plazo.

Las acciones de mayor urgencia incluyen, en primer lugar, priorizar la vacunación de los sectores de la economía del cuidado (salud, enseñanza y trabajo doméstico remunerado), reconociéndolos como pilar fundamental de la sostenibilidad de la vida y de la recuperación. En segundo lugar, es necesario fortalecer los ingresos de las mujeres a través del acceso a un ingreso básico de emergencia, la entrega de alimentos e insumos básicos en el contexto de la emergencia sanitaria, así como la ampliación o creación de nuevas líneas de financiamiento dirigidas a mujeres en micro, pequeñas y medianas empresas. En tercer lugar, se deben garantizar los derechos de las personas que cuidan y la atención de quienes deben ser cuidados. Para esto, medidas como los permisos de desplazamiento por cuidado, licencias especiales para realizar tareas de cuidado y las campañas sobre la corresponsabilidad en los cuidados, se vuelven centrales a la hora de visibilizar y redistribuir este trabajo.

Por otra parte, es necesario generar acciones concretas e inmediatas para otros sectores altamente feminizados que han sido especialmente afectados por la pandemia, tales como el comercio, el turismo, la industria manufacturera y en sectores de la economía del cuidado (especialmente, servicio doméstico). Particularmente en el sector del trabajo doméstico remunerado, ya en algunos países se han generado medidas de emergencia, entre ellas, su inclusión como población objetivo en las transferencias monetarias de emergencia por la pandemia de COVID-19.

Sin embargo, a pesar de ser sumamente necesarias, estas medidas de corto plazo no son suficientes. Es imprescindible diseñar e implementar medidas con orientación transformativa, de mediano y de largo plazo en el ámbito de la fiscalidad, el empleo, las políticas productivas, económicas y sociales, que fomenten la autonomía económica de las mujeres y protejan sus derechos.

En primer lugar, es necesario promover un pacto fiscal que promueva la igualdad, lo que supone la generación de análisis acerca de los efectos diferenciados por género de las medidas fiscales impulsadas, así como un aumento de los niveles de recaudación de forma progresiva y asignación de gasto hacia políticas de género en la emergencia y a largo plazo. El financiamiento de los servicios esenciales como los servicios de atención a situaciones de violencia por razón de género, los servicios de salud sexual y reproductiva, los servicios de cuidado, y mecanismos de protección de los ingresos para las mujeres debe ser prioritario. Además, es necesario promover medidas de estímulo fiscal orientadas a proteger los ingresos y el empleo de las mujeres, incluyendo medidas afirmativas para el acceso a empresas de mujeres a las contrataciones y compras públicas e inversión en sectores dinamizadores con enfoque de género. Por último, se requiere fortalecer la cooperación regional y global para movilizar recursos adicionales a nivel internacional y que los países de renta media de la región puedan acceder al financiamiento internacional en condiciones favorables.

En segundo lugar, es necesario implementar acciones estatales orientadas a impactar en las transformaciones del mundo del trabajo que han sido catalizadas por la actual pandemia, buscando además evitar la precarización de las formas de empleo emergentes. Esto se ha vuelto especialmente notorio en lo que se refiere al empleo digital y de plataformas. Por esto, se debe fomentar una transformación digital incluyente, garantizando los derechos de las mujeres al trabajo y en el trabajo. Para ello, se deben impulsar acciones orientadas a la protección de los derechos laborales, así como de la generación de una canasta básica digital que potencie las habilidades para utilizar dichas tecnologías, reconociendo los nuevos requerimientos para la plena participación en la educación y en el trabajo. 
En tercer lugar, es necesario impulsar una recuperación productiva con empleo para las mujeres a través de la acción deliberada y explícita de parte de las instituciones públicas - a diferentes escalas - por estimular aquellos sectores que afectan especialmente a la autonomía económica de las mujeres. En esta línea, se deberán buscar sinergias positivas con sectores que promuevan marcos económicos sostenibles, tales como el turismo sostenible, las energías renovables, la economía circular, la industria manufacturera de la salud, la economía digital y la bioeconomía.

Finalmente, es indispensable impulsar el potencial transformativo de la economía del cuidado como un elemento central hacia una recuperación transformadora con igualdad. En este sentido, se deben generar acciones orientadas a la creación y fortalecimiento de los sistemas integrales del cuidado; articulando políticas de distribución del tiempo, recursos, prestaciones y servicios, y buscando promover el principio de corresponsabilidad entre hombres y mujeres, así como el Estado, el mercado, las familias y comunidades.

Es necesario modificar el abordaje de los cuidados desde una perspectiva que considere este sector, exclusivamente, como un gasto hacia una aproximación que lo conceptualice también como una inversión. El fortalecimiento de la economía del cuidado debe ser comprendido como una inversión en términos de capacidades presentes y futuras, así como de generación de empleo, en particular para las mujeres. Además, de articularse con políticas laborales que mejoren la calidad de esos empleos, el sector de los cuidados puede contribuir a revertir las discriminaciones en el mercado laboral a través de la mejora en las condiciones en este sector. A mediano y largo plazo, la inversión en la economía del cuidado aumenta también la productividad y los niveles de recaudación. Todo ello constituye a la economía del cuidado como un área clave para la recuperación sostenible y con mayor igualdad.

Los factores que han exacerbado los efectos de la pandemia constituyen elementos de largo plazo a nivel regional. Por ello, la respuesta a la crisis sanitaria, económica y social debe acompañarse de acciones que busquen, en simultáneo, generar los cimientos para modificar el modelo de desarrollo actual. La crisis se presenta, así, como una oportunidad para el cambio hacia sociedades más sostenibles, justas e igualitarias.

Esta es la oportunidad de desatar los nudos estructurales de la desigualdad que atentan contra la autonomía económica, física y política de las mujeres, y construir sociedades de cuidado para un futuro más igualitario, sostenible y resiliente. Transitar hacia una sociedad del cuidado, en la que cuidemos al planeta, a las personas y a quienes cuidan, así como se promueva el autocuidado implica repensar los patrones de producción, consumo, distribución, y reorientar las economías a lo que realmente es importante y valioso: la sostenibilidad de la vida.

\section{Referencias bibliográficas}

Bidegain Ponte, N., Scuro Somma, L., \& Vaca-Trigo, I. (2020). La autonomía económica de las mujeres en tiempos de COVID-19. Revista CEPAL. LC/PUB.2021/4-P.

Braunstein, E., van Staveren, I., \& Tavani, D. (2011). Embedding care and unpaid work in macroeconomic modeling: a structuralist approach. Feminist Economics, 17(4), 5-31. Taylor and Francis.

CEPAL, Comisión Económica para América Latina y el Caribe. (2017, marzo). Estrategia de Montevideo para la Implementación de la Agenda Regional de Género en el Marco del Desarrollo Sostenible hacia 2030. (LC/ CRM.13/5). Santiago.

CEPAL, Comisión Económica para América Latina y el Caribe. (2019a). Balance Preliminar de las Economías de América Latina y el Caribe, 2019. (LC/PUB.2019/25-P). Santiago.

CEPAL, Comisión Económica para América Latina y el Caribe. (2019b, diciembre). La autonomía de las mujeres en escenarios económicos cambiantes. (LC/CRM.14/3). Santiago.

CEPAL, Comisión Económica para América Latina y el Caribe. (2020a). Balance Preliminar de las Economías de América Latina y el Caribe, 2020. (LC/PUB.2020/17-P). Santiago.

CEPAL, Comisión Económica para América Latina y el Caribe. (2020b, abril). La pandemia del COVID-19 profundiza la crisis de los cuidados en América Latina y el Caribe. Informes COVID-19. N. ${ }^{\circ} 1$.

CEPAL, Comisión Económica para América Latina y el Caribe. (2021a). La autonomía económica de las mujeres en la 
recuperación sostenible y con igualdad. Informe Especial COVID-19. N. ${ }^{\circ}$ 9. Santiago.

CEPAL, Comisión Económica para América Latina y el Caribe. (2021b). Panorama Social de América Latina, 2020. (LC/ PUB.2021/2-P/Rev.1). Santiago, 2021. Página 211.

CEPAL, Comisión Económica para América Latina y el Caribe. (2021c). Construir un futuro mejor: acciones para fortalecer la Agenda 2030 para el Desarrollo Sostenible. (LC/FDS.4/3/Rev.1). Santiago, 2021. Página 181.

CEPAL/OIT, Comisión Económica para América Latina y el Caribe/Organización Internacional del Trabajo. (2020, noviembre). La dinámica laboral en una crisis de características inéditas: desafíos de política. Coyuntura Laboral en América Latina y el Caribe, N. ${ }^{\circ}$ 23. (LC/ TS.2020/128). Santiago.

OIT, Organización Internacional del Trabajo. (2016, agosto). La migración laboral en América Latina y el Caribe. Diagnóstico, estrategia y líneas de trabajo de la OIT en la Región. Lima, Oficina Regional para América Latina y el Caribe. Ginebra.
OIT, Organización Internacional del Trabajo. (2018). Care Work and Care Jobs for the Future of Decent Work. Ginebra.

OIT, Organización Internacional del Trabajo. (2020). COVID-19 and the world of work. Fifth edition. Updated estimates and analysis. ILO Monitor. https://www.ilo.org/wcmsp5/groups/ public/@dgreports/@dcomm/documents/briefingnote/ wcms_749399.pdf

Vaca-Trigo, I. (2019, enero). Oportunidades y desafíos para la autonomía de las mujeres en el futuro escenario del trabajo. Serie Asuntos de Género, N. ${ }^{\circ} 154$ (LC/TS.2019/3). Santiago. Comisión Económica para América Latina y el Caribe (CEPAL).

Valenzuela, M. E., Scuro Somma, L., \& Vaca-Trigo, I. (2020). Desigualdad, crisis de los cuidados y migración del trabajo doméstico remunerado en América Latina. Serie Asuntos de Género, N. ${ }^{\circ}$ 158. (LC/TS.2020/179). Santiago. Comisión Económica para América Latina y el Caribe (CEPAL), página 85. 